\title{
Safety and efficacy of treatment of very small intracranial aneurysms
}

\author{
Tomasz Jamróz ${ }^{1 A, B, C, D, E}$, Izabela Jakutowicz ${ }^{1 A, B, C, D, E}$, Mariusz Hofman ${ }^{2 A, B, C, D, E}$, Marta Kołodkiewicz ${ }^{1 B, F}$, \\ Maciej Ćmiel ${ }^{1 B, F}$, Anna Łapaj ${ }^{1 B, F}$, Nikodem Przybyłko ${ }^{30, G}$, Piotr Bażowski10,G, Jan Baron ${ }^{10, G}$ \\ 'School of Medicine in Katowice, Medical University of Silesia, Katowice, Poland \\ 2Department of Anaesthesiology and Intensive Care, School of Medicine in Katowice, Medical University of Silesia, Katowice, Poland \\ ${ }^{3}$ Department of Neurosurgery, School of Medicine in Katowice, Medical University of Silesia, Katowice, Poland
}

\section{Abstract}

\begin{abstract}
Purpose: Very small intracranial aneurysms (VSIAs) may cause many neurological complications and even death. Thanks to technological progress and higher quality of non-invasive neuroimaging methods, these pathologies can be investigated sooner and treated earlier. Due to the controversy surrounding invasive treatment of these pathologies, the aim of the study was to analyse methods of treatment, their outcome, and complications in a group of patients with VSIAs.

Material and methods: Out of 444 cases of intracranial aneurysms treated in our centre, 65 aneurysms met the radiological criteria of VSIAs. The parameters - width and length of the aneurysm's neck and width, length, and height of the aneurysm's dome - were measured. The analysed parameters were as follows: symptoms upon admission and after treatment, days in hospital, and intraoperative complications. Clinical and radiological intensity of subarachnoid haemorrhage (SAH) was evaluated by using the Hunt-Hess and Fisher scales. The degree of embolisation of the aneurysm after the procedure was assessed using the Montreal Scale. Clinical outcome was assessed by Glasgow Outcome Scale.
\end{abstract}

Results: $50.77 \%$ of VSIAs were treated with endovascular procedures and $49.23 \%$ with neurosurgical clipping. SAH was presented in $38.46 \%$ of patients with VSIAs. Intraoperative complications were presented in $16.92 \%$ of patients with VSIAs, and the most common complication was ischaemic stroke. Stents were used in $51.52 \%$ of VSIAs. In $69.70 \%$ of embolisation procedures at VSIAs complete obliteration was achieved. The average result in the Montreal Scale was $1.31(\mathrm{SD}=0.66)$.

Conclusion: VSIAs can be treated as effectively and safely as larger aneurysms, by both endovascular and surgical methods.

Key words: intracranial aneurysm, endovascular procedures, subarachnoid haemorrhage.

\section{Introduction}

Recently, technological progress, better availability, and higher quality of non-invasive neuroimaging is remarkable. Pathologies with smaller measurements can be investigated and treated earlier. Thanks to that we can extract very small intracranial aneurysms (VSIAs) from the group of intracranial aneurysms. It is crucial to diagnose aneurysms before they burst, to avoid or minimise neurological complications and death. Because unruptured intracranial aneurysms create a risk of subarachnoid haemorrhage (SAH), risk factors of rupturing such as sex, age, smoking, hypertension, aspect ratio, size, irregular shape, and location at bifurcation or posterior circulation should be considered [1].

The aim of our study was to analyse methods of treatment, their outcome, and complications in a group of patients with VSIAs.

\section{Material and methods}

For our study, we retrospectively reviewed collected data of all patients diagnosed with intracranial aneurysms in

Correspondence address:

Tomasz Jamróz, School of Medicine in Katowice, Medical University of Silesia, 18 Medyków St., 40-752 Katowice, Poland, e-mail: Tomek143@gmail.com

Authors' contribution:

A Study design · B Data collection · C Statistical analysis · D Data interpretation · E Manuscript preparation · F Literature search · G Funds collection 
our centre in the years 2008-2014. In those years the number of patients, treated both invasively and conservatively, gradually increased up to 300 cases per year in 2014. During these years, coil embolisation was the main treatment method in our centre. In cases of wide-neck aneurysms stent-assisted coiling was always considered. Flow-diverters have not been used in our centre yet. Only the first procedure regarding particular aneurysms was taken into consideration during data analysis. Patients who did not undergo digital subtraction angiography (DSA) were excluded from this study. All patients who were not qualified to invasive treatment, including patients with VSIA, were also excluded. In total 444 patients met the inclusion criteria and were qualified to this study.

All aneurysms were visualised using 3-dimensional DSA (3D-DSA) on a Philips Integris Allura. Then, using $3 \mathrm{D}$ reconstruction, the aneurysm domes and necks were

Table 1. Calculations performed to obtain several options for bottleneck (BN) factors

\begin{tabular}{|l|l|}
\hline BN minimal & Minimal dome dimension/Maximal neck dimension \\
\hline BN maximal & Maximal dome dimension/Minimal neck dimension \\
\hline BN width & Dome width/Neck width \\
\hline BN length & Dome length/Neck length \\
\hline BN average & $\begin{array}{l}\text { Arithmetical average of BN minimal, BN maximal, } \\
\text { BN width, BN length }\end{array}$ \\
\hline
\end{tabular}

Table 2. Comparison of bottleneck (BN) factors and average size among very small intracranial aneurysms (VSIAs) and the control group

\begin{tabular}{|l|c|c|c|}
\hline Mean size & VSIAs & Control group & $p$ \\
\hline BN minimal & 0.84 & 1.22 & 0.000001 \\
\hline BN maximal & 1.39 & 1.91 & 0.000001 \\
\hline BN width & 1.13 & 1.62 & 0.000001 \\
\hline BN length & 1.01 & 1.47 & 0.000001 \\
\hline BN average & 1.11 & 1.57 & 0.000001 \\
\hline Average size & 3.26 & 7.06 & 0.000001 \\
\hline
\end{tabular}

Table 3. Intraoperative complications

\begin{tabular}{|l|c|c|}
\hline Complication & $\begin{array}{c}\text { Very small } \\
\text { intracranial } \\
\text { aneurysms }\end{array}$ & $\begin{array}{c}\text { Control } \\
\text { group }\end{array}$ \\
\hline $\begin{array}{l}\text { Bleeding from aneurysm } \\
\text { during procedure }\end{array}$ & $1(1.54 \%)$ & $12(3.17 \%)$ \\
\hline Intracranial haematoma & $1(1.54 \%)$ & $11(2.90 \%)$ \\
\hline Wound infection & $2(3.08 \%)$ & $5(1.32 \%)$ \\
\hline Ischemic stroke & $3(4.61 \%)$ & $14(3.69 \%)$ \\
\hline Injection site haematoma & $2(3.08 \%)$ & $9(2.37 \%)$ \\
\hline Brain oedema & $1(1.54 \%)$ & $0(0.00 \%)$ \\
\hline Liquorrhoea & $1(1.54 \%)$ & $3(0.79 \%)$ \\
\hline Pneumocephalus & $0(0.00 \%)$ & $1(0.26 \%)$ \\
\hline
\end{tabular}

measured. The following parameters were obtained: width and length of the aneurysm neck; width, length, and height of the aneurysm dome. Bottleneck factors (BN) were counted in several combinations (Tables 1 and 2). The average size was calculated as the arithmetic average from all dome parameters.

A cohort of 65 cases, representing $14.64 \%$ of the total population, met our criteria of VSIA, which were any measured dome dimension $\leq 3 \mathrm{~mm}$. The group included 43 women and 22 men, with an average age of 53.28 $(\mathrm{SD}=11.31)$ years. The remaining 379 cases $(85.36 \%)$ were classified into a control group.

The analysed parameters were as follows: symptoms presented upon admission and their withdrawal after treatment, days spent in the Neurosurgery Department and Intensive Care Unit, and intraoperative complications. SAH was assessed using the Hunt and Hess scale and original Fisher scale [2-4].

For assessment of the immediate and long-term outcomes of endovascular procedures, residual patency rates on the three-item score of the Montreal scale $(1=$ total occlusion, 2 = neck remnant, and 3 = aneurysm remnant) were registered directly after embolisation and in follow-ups [5]. Neurosurgical treatment was assessed respectively based on completeness of clipping. Follow-up examinations were carried out at least six months after the procedure and employed either DSA (27 patients, $40.9 \%$ ) or CT angiography (four patients, 6.1\%). Upon patient discharge the Glasgow Outcome Scale (GOS) scores were assessed ( 1 - death, 2 - persistent vegetative state, 3 - severe disability, 4 - moderate disability, 5 - low or no disability) [6].

All statistical analysis was performed using Statistica 12 software. Due to non-normal distribution, all analysis was performed using Mann-Whitney $U$ test, Kruskal-Wallis ANOVA, and McNemar's test. IRB approval for this study, as well as for any retrospective study in our university, is not required by the Institution Ethical Committee.

\section{Results}

Among 65 VSIAs, 33 endovascular procedures (50.77\%) were carried out, including $24(72.73 \%)$ cases of unruptured and nine (27.27\%) cases of ruptured aneurysms. Neurosurgical clipping was employed in 32 cases (49.23\%), with $16(50.00 \%)$ patients presenting SAH and 16 (50.00\%) patients with no intracranial bleeding. Distribution of sex, age, and the presence of SAH in our study group corresponded to all analysed cerebral aneurysms.

Predominant locations of aneurysms in our study group were anterior communicating artery (36.92\%), followed by middle cerebral artery (32.31\%) (Figure 1). Mean size is presented in Table 4.

Upon admission to the hospital, SAH was presented in $25(38.46 \%)$ patients with VSIA and 124 (32.72\%) patients in the control group $(p>0.05)$. 
Intraoperative complications were presented more often in patients with VSIA than in patients in the control group (16.92\% vs. $13.19 \%)$ but without statistical significance $(p=0.42)$. Table 3 outlines noted complications. Considering each particular complication between groups, statistical significance was only discovered in brain oedema prevalence $(p=0.02)$. However, that result is probably caused by the existence of only one case. The most common complication in both groups was ischaemic stroke.

Stents were used in 17 cases (51.52\%) of the very small aneurysm group. In the control group, they were used in 137 patients (50.74\%).

Table 4 illustrates Montreal Scale results. In 25 cases, including procedures in the VSIA and control group, the Montreal Scale results were not assessed due to interruption of the procedure. In another two cases it was not possible to assess the Montreal score retrospectively. In $69.70 \%$ of embolisation procedures at VSIA complete obliteration was achieved. The average Montreal Scale result was $1.31(\mathrm{SD}=0.66)$. Due to technical difficulties, intervention failed in four (12.12\%) cases. Twice the failure was caused by drug-resistant vasospasm. In one case the coil evacuated from the aneurysm sack. In the last patient the coil could not be placed into the sac of the aneurysm despite numerous attempts. In the control group, average Montreal Scale score was $1.37(\mathrm{SD}=0.64)$ and did not differ with VSIA group $(p=0.44)$. Of the $21(7.78 \%)$ interrupted embolisations in the control group: three times the aneurysm ruptured, drug resistant vasospasm caused a failure eight times, and complications with insertion of coils into the aneurysm sac resulted in seven failed procedures. Information about the remaining three cases could not be retrospectively obtained.

All neurosurgical procedures in both groups of patients led to complete isolation from circulation.

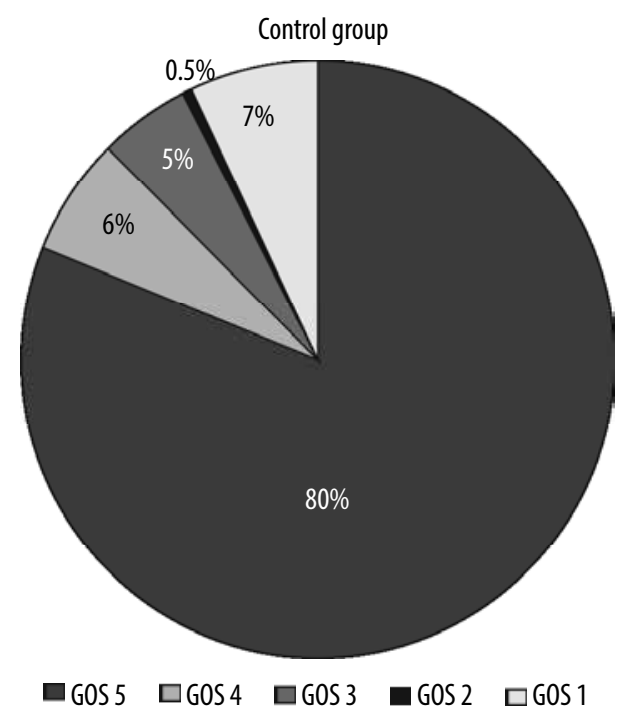

Figure 2. Patient outcomes in the Glasgow Outcome Scale (GOS)

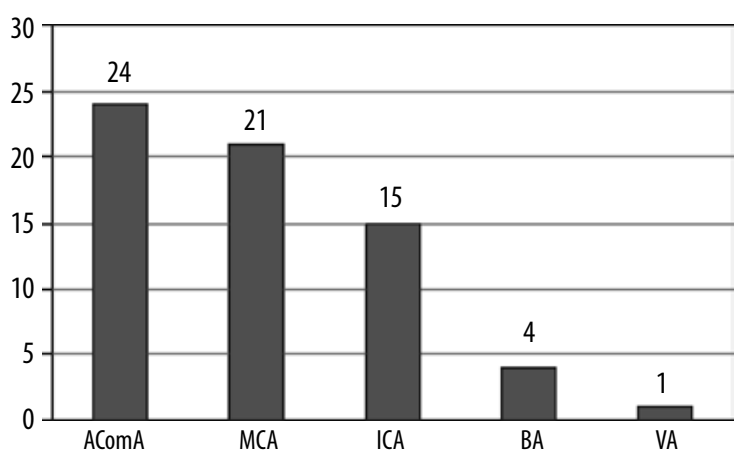

AComA - anterior communicating artery, MCA - middle cerebral artery, ICA - internal carotid artery, BA - basilar artery, $\mathrm{VA}$ - vertebral artery

Figure 1. Distribution of very small intracranial aneurysms

Table 4. Efficacy of endovascular procedures using the Montreal Scale

\begin{tabular}{|l|c|c|}
\hline Montreal Scale score & $\begin{array}{c}\text { Very small } \\
\text { intracranial } \\
\text { aneurysms }\end{array}$ & $\begin{array}{c}\text { Control } \\
\text { group }\end{array}$ \\
\hline I & $23(69.70 \%)$ & $176(65.43 \%)$ \\
\hline II & $3(9.09 \%)$ & $51(18.96 \%)$ \\
\hline
\end{tabular}

The average hospitalisation period of patients in the VSIA group was 15 days, including 11 days in the Neurosurgery Department and four days in the Intensive Care Unit. In the control group the results were 10, eight, and two days, respectively. Differences were not statistically significant ( $p=0.44, p=0.52$, and $p=0.36$, respectively).

$178(58.75 \%)$ patients who underwent endovascular embolisation had arrived at a follow-up examination, including 21 patients (63.64\%) with VSIA. Average results in the Montreal Scale differ between the VSIA (1.10, $\mathrm{SD}=0.30)$ and control groups $(1.38, \mathrm{SD}=0.67)$, but there is no statistical significance $(p=0.07)$.

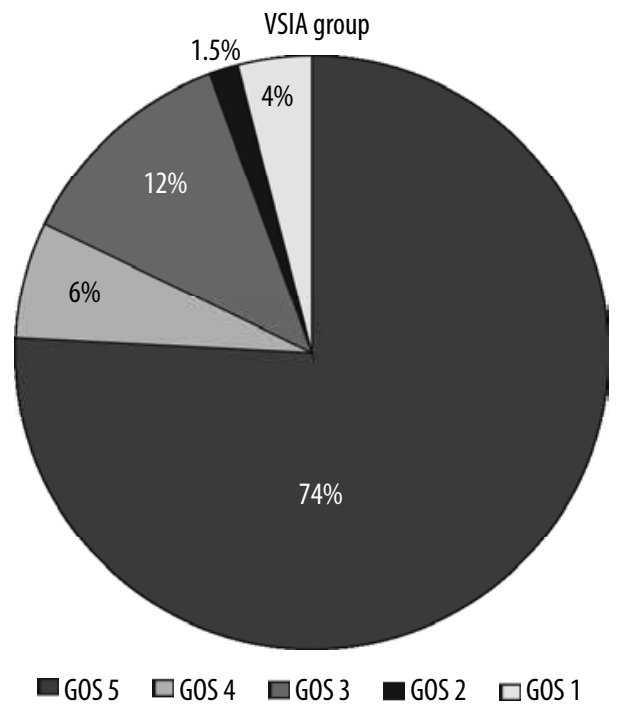


Mean Glasgow Outcome Scale scores were 4.45 (SD $=1.08)$ and $4.54(\mathrm{SD}=1.12)$ for the VSIA and control groups, respectively, with no statistically significant difference $(p=0.24)$. As had been presented in Figure 2, the majority of patients recovered well after the procedure. There was nonsignificant $(p=0.45)$ dissimilarity in mortality. Thirty patients in our study died: three $(4.62 \%)$ in the VSIA group and $27(7.12 \%)$ in the control group.

\section{Discussion}

In the literature, very small intracranial aneurysms are defined as intracranial aneurysms with a diameter smaller than or equal to $3 \mathrm{~mm}$ [7-9]. Other sources qualify these aneurysms to a group called "baby aneurysms" [10,11]. Unfortunately, the sources do not indicate which dimension is analysed in qualification of the aneurysm into the VSIA group; therefore, in our study, to the VSIA group we qualified aneurysms with any dimension less than $3 \mathrm{~mm}$. Based on our criteria we qualified 65 patients with VSIA for the study.

Analysing the therapeutic problems thast VSIA can cause, therapeutic decisions should be individual for each patient $[12,13]$. Keeping in mind patients' health and considering the possible complications, we should choose the best treatment from the following invasive methods: neurosurgical clipping, endovascular coiling, or by-pass surgery [14-16]. In our centre two of those methods of treatment are being performed: surgery clipping and endovascular procedure. As by-pass surgery is not an available option, we cannot analyse the effectiveness of this method in aneurysm treatment. We realise that this is a restriction for our research. In the literature there are not many articles comparing endovascular and neurosurgery treatment for very small aneurysms $[12,13]$. Therefore, in our study, we decided to analyse treatment methods, complications, and mortality with regard to the control group of patients with larger aneurysms.

In patients with VSIA there are many factors that can cause technical and therapeutic problems. In view of their small size, the VSIA are a therapeutic problem for neurosurgeons and invasive radiologists. The small size of the aneurysm restricts the freedom of the operator's movement, and it increases the risk of uncontrolled dislocation of the catheter and microaneurysm rupture. Additionally, in very small aneurysm there is greater risk of putting the microcatheter too close to the primordial site of the rupture [17-19]. In our centre, due to technical problems, $12.12 \%$ of treatment procedures have failed. In the literature there are also data about interrupted operations due to technical problems [13]. Other authors mention coil-related problems, but they do not specifically indi- cate what the problems are and how often they occur [20]. In the literature there is no definite position that would indicate the frequency of occurrence of technical problems during the procedure and what complications should be included in this group. Accordingly, we cannot clearly compare our results with other publications. However, we can compare these results between the VSIA group with the control group, where there is no statistical significance.

In our centre the most common complication was ischaemic stroke. It was also described as a complication of treatment in other centres, but it was not the most common $[21,22]$. Data show that frequent ischaemic events were related to patient age and other adverse factors. Other complications seen in our centre such as rupture of the aneurysm during surgery are also described in other publications. It was $1.54 \%$ in our centre, while in the literature it is described as $3.92 \%$ to $4.90 \%$ [10,20,23]. Some authors describe how VSIA rupture associated with procedure occurs twice or even five times more than in larger aneurysms $[17,24,25]$. On the other hand, in other sources the authors reported that during the procedure there were no complications [24,26].

In our study we checked bottleneck factors, and our analysis showed that VSIA have lower values of bottleneck factors then aneurysms in the control group. In connection, we could expect more complications in this group of patients than in the control group [22]. However, in our study the complication ratio was comparable in both groups. The time of hospitalisation was similar for patients with VSIA and patients with other intracranial aneurysms investigated in our study. Finally, there were no differences for these groups and expectedly the mortality was not higher in the group of patients with VSIA. Our mortality outcomes are comparable with data in the literature [27].

\section{Conclusions}

In conclusion, the criteria used in this study to isolate patients with VSIA from patients with other intracranial aneurysms may help those patients who need more precautions in the therapeutic process. In addition, the development of techniques used in the treatment of aneurysms allows the safe treatment of minor changes by both endovascular and surgical methods. It appears that these aneurysms can be treated as effectively and safely as larger aneurysms. This topic requires further investigation.

\section{Conflict of interest}

The authors report no conflict of interest. 


\section{References}

1. Feng X, Ji W, Qian Z, et al. Bifurcation location is significantly associated with rupture of small intracranial aneurysms $(<5 \mathrm{~mm})$. World Neurosurg 2017; 98: 538-545.

2. Mascitelli JR, Moyle H, Oermann EK, et al. An update to the Raymond-Roy Occlusion Classification of intracranial aneurysms treated with coil embolization. J Neurointerv Surg 2015; 7: 496-502.

3. Jennett B, Bond M. Assessment of outcome after severe brain damage: a practical scale. Lancet 1975; 1: 480-484.

4. Hunt WE, Hess RM. Surgical risk as related to time of intervention in the repair of intracranial aneurysms. J Neurosurg 1968; 28: 14-20.

5. Fisher CM, Kistler JP, Davis JM. Relation of cerebral vasospasm to subarachnoid hemorrhage visualized by CT scanning. Neurosurgery 1980; 6: 1-9.

6. Frontera JA, Claassen J, Schmidt JM, et al. Prediction of symptomatic vasospasm after subarachinoid hemorrhage: the modified Fisher scale. Neurosurgery 2006; 59: 21-27.

7. Rahmanian A, Ghaffarpasand F, Derakhshan N. Surgical outcome of patients with very small intracranial aneurysms; a single center experience from Southern Iran. World Neurosurg 2017; 98: 470-478.

8. Yamaki VN, Brinjikji W, Murad MH, et al. Endovascular treatment of very small intracranial aneurysms: meta-analysis. Am J Neuroradiol 2016; 37: 862-867.

9. Brinjikji W, Lanzino G, Cloft HJ, et al. Endovascular treatment of very small ( $3 \mathrm{~mm}$ or smaller) intracranial aneurysms. report of a consecutive series and a meta-analysis. Stroke 2010; 41: 116-121.

10. Ioannidis I, Lalloo S, Corkill R, et al. Endovascular treatment of very small intracranial aneurysms. J Neurosurg 2010; 112: 551-556.

11. Yaşargil MG. Pathological considerations. In: Microneurosurgery. Stuttgart: Thieme Medical Publishers Inc; 1987, 280-281.

12. Li J, Su L, Ma J, et al. Endovascular coiling versus microsurgical clipping for patients with ruptured very small intracranial aneurysms: Management strategies and clinical outcomes - of 162 cases. World Neurosurg 2017; 99: 763-769.

13. Chalouhi N, Penn DL, Tjoumakaris S, et al. Treatment of small ruptured intracranial aneurysms: comparison of surgical and endovascular options. J Am Heart Assoc 2012; 1: e002865.

14. Bruneau M, Amin-Hanjani S, Koroknay-Pal P, et al. Surgical clipping of very small unruptured intracranial aneurysms: a multicenter international study. Neurosurgery 2015; 78: 47-52.
15. Iskandar A, Nepper-Rasmussen J. Endovascular treatment of very small intracranial aneurysms. Interv Neuroradiol 2011; 17: 299-305.

16. Bonda DJ, Labib M, Katz JM, et al. Intracranial bypass of posterior inferior cerebellar artery aneurysms: indications, technical aspects, and clinical outcomes. Operative Neurosurgery 2017; 13: 586-595.

17. Nguyen TN, Raymond J, Guilbert F, et al. Association of endovascular therapy of very small ruptured aneurysms with higher rates of procedure-related rupture. J Neurosurg 2008; 108: 1088-1092.

18. Sluzewski M, Bosch JA, van Rooij WJ, et al. Rupture of intracranial aneurysms during treatment with Guglielmi detachable coils: incidence, outcome, and risk factors. J Neurosurg 2001; 94: 238-240.

19. Cloft HJ, Kallmes DF. Cerebral aneurysm perforations complicating therapy with Guglielmi detachable coils: a meta-analysis. AJNR Am J Neuroradiol 2002; 23: 1706-1709.

20. Pierot L, Barbe C, Spelle L, et al. Endovascular treatment of very small unruptured aneurysms rate of procedural complications, clinical outcome, and anatomical results. Stroke 2010; 41: 2855-2859.

21. Qin F, Li Z, Fang X, et al. Therapeutic effect of enterprise stentassisted embolization for very small ruptured intracranial aneurysms. Medicine (Baltimore) 2017; 96: e7832.

22. Hwang SK, Hwang G, Bang JS, et al. Endovascular Enterprise stent-assisted coil embolization for wide-necked unruptured intracranial aneurysms. J Clin Neurosci 2013; 20: 1276-1279.

23. Liu Y, Wang F, Fu X, et al. Clinical and angiographic outcomes following endovascular treatment of very small ( $3 \mathrm{~mm}$ or smaller) intracranial aneurysm. A single-center experience. Medicine (Baltimore) 2017; 96: e7457.

24. Hang JH, Roh HG, Chun YI, et al. Endovascular coil embolization of very small intracranial aneurysms. Neuroradiology 2011; 53: 349-357.

25. van Rooij WJ, Keeren GJ, Peluso JPP, et al. Clinical and angiographic results of coiling of 196 very small $(\leq 3 \mathrm{~mm})$ intracranial aneurysms. Am J Neuroradiol 2009; 30: 835-839.

26. Suzuki S, Kurata A, Ohmomo T, et al. Endovascular surgery for very small ruptured intracranial aneurysms. J Neurosurg 2006; 105: 777-780.

27. Chae KS, Jeon P, Kim KH, et al. Endovascular coil embolization of very small intracranial aneurysms. Korean J Radiol 2010; 11: 536-541. 